Arnold Schönbergs

Verhältnis zur populären Musik:

Operette, Vokal- und Instrumentalmusik

\author{
Peter Andraschke † \\ Univerza Justusa Liebiga v Giessnu (JLU) \\ Justus-Liebig-Universität Gießen
}

Arnold Schönberg (1874, Wien - 1951, Los Angeles) ist einer der bedeutendsten Schöpfer der Neuen Musik im 2o. Jahrhundert, seine Musik hat ihren festen Platz in den Medien und wird von der Wissenschaft diskutiert. Er stammt aus einer musikinteressierten Familie, erhielt seit seinem 8. Lebensjahr gemeinsam mit seiner Schwester Geigenunterricht und besaß seit seiner Kindheit ein selbstverständliches Verhältnis zur leichten Muse, mit der er aufgewachsen ist. Sie war für ihn als Autodidakt wichtig für seine musikalische Bildung, wie das bekannte Zitat festhält, in dem er erzählt, zu Hause wären trotz aller Förderung

die Zeichen von musikalischem Talent, die bei mir erschienen, nicht ernstlich beachtet [worden]. Deshalb sind alle Kompositionen, die ich vor meinem siebzehnten Jahr geschrieben habe, nichts als Imitationen solcher Musik, die mir zugänglich war. Die einzigen Quellen, aus denen ich schöpfen konnte, waren Violinduette und Arrangements von Opernpotpourris für zwei Violinen, wozu noch die Musik gerechnet werden darf, die ich durch Militärkapellen kennenlernte, die in öffentlichen Gärten Konzerte gaben. Man darf übrigens nicht vergessen, daß zu dieser Zeit Noten sehr teuer waren, daß es weder

$1 \quad$ Peter Andraschke hat seinen Artikel knapp vor seinem Tod als Vortragsmanuskript fertiggestellt und die Zusammenfassung bereits nach Ljubljana gemailt. Der Artikel selbst konnte in Wien in seinem Computer sichergestellt werden. Hartmut Krones hat ihn für den Druck eingerichtet sowie die Fußnoten ergänzt und mit einer inhaltlichen Anmerkung versehen. 
Platten noch Radio gab, und daß Wien nur ein einziges Opernhaus und einen einzigen Zyklus von philharmonischen Konzerten hatte. ${ }^{2}$

$\mathrm{Zu}$ den frühen Kompositionsversuchen Schönbergs gehören das ländlerartige Stück für Violine und Klavier (1893/94) und die zehn Walzer für Streichorchester ${ }^{4}$. Es sind abwechslungsreiche charmante Stücke, die sich im Ton neben dem Wiener Walzer der Straußdynastie zuweilen auch an anderen Vorbildern orientieren, z. B. an Antonín Dvořák und Franz Schubert.

Die zahlreichen biographischen Nachrichten, die Schönbergs Verhältnis zur unterhaltenden Musik belegen, überraschen nicht. Sie zeigen, daß Schönberg ein Wiener Kind seiner Zeit war. Eine derartige soziale Kluft, wie sie sich im Laufe des 20. Jahrhunderts zwischen den Hörern der sogenannten E- sowie der U-Musik herausgebildet hat, war im Wien der Jahrhundertwende noch nicht vorhanden.

Zwischen 1900 und 1904 arrangierte und instrumentierte Schönberg, um seinen Lebensunterhalt zu finanzieren, unzählige Werke anderer Komponisten, vor allem Operetten von sehr unterschiedlicher Qualität. Es waren insgesamt rund 6000 Seiten. ${ }^{6}$ In diesen Jahren entstanden bereits seine opera 1-6, darunter die Verklärte Nacht für Streichsextett op. 4, Pelleas und Melisande op. 5 und die Anfänge der Gurrelieder. Die Operettenarbeiten betrafen auch Komponisten und Werke, die damals erfolgreich waren und noch in der Zwischenkriegszeit gespielt wurden, u. a. von Adalbert v. Goldschmidt (1848-1906) und Bruno Granichstaedten (1879-1944), später in Berlin war Bogumil Zepler (1858-1918) der größte Auftraggeber. Aus den persönlichen Kontakten entwickelten sich Freundschaften, die ihm Kontakte für sein Engagement als Dirigent an Ernst von Wolzogens Berliner Kabarett Überbrettl brachten, wo bereits Edmund Eysler und Oscar Straus tätig waren. Anfang 1901 hatte er seine Brettl-Lieder komponiert, deren Nummern durchaus in Operetten stehen könnten. Sie überzeugten Wol-

2 Arnold Schönberg, „Rückblick“, in Stil und Gedanke. Aufsätze zur Musik (Gesammelte Schriften 1), Hrsg. Ivan Vojtěch (Frankfurt a. M.: Fischer, 1976), 397.

3 Copyright 2001 by Belmont Music Publishers, Pacific Palisades.

4 Copyright 2003 by Belmont Music Publishers, Los Angeles. Ein elfter Walzer blieb Fragment.

5 Therese Muxeneder, „Walzer für Streichorchester (ca. 1897). Einführung“, in Salzburger Festspiele 2004. Programmheft der Uraufführung (Salzburg: s. n., August 2004).

6 H. H. Stuckenschmidt, Schönberg. Leben. Umwelt. Werk (Zürich und Freiburg: Atlantis, 1974), 48. 
zogen anläßlich eines Gastspiels am Wiener Carltheater ${ }^{7}$, das Schönbergs Schwager Alexander Zemlinsky sechs Jahre leitete und dort vor allem Operetten dirigierte.

1903 kehrte Schönberg nach eineinhalbjährigem Berliner Aufenthalt, wo sich seine beruflichen Hoffnungen zerschlagen hatten, nach Mödling zurück und mußte wieder die Instrumentierung von Werken anderer aufnehmen. Es existiert ein Brief an Karl C. Posa (1873-1951), mit dem sowie mit Alexander Zemlinsky er 1904 die Vereinigung schaffender Tonkünstler in Wien gegründet hatte, auch zum Eigenzweck. Sie sollte laut Statuten die „Pflege und Förderung der Werke der zeitgenössischen Tonkunst“ sowie „die Wahrung der künstlerischen und Standesinteressen ihrer Mitglieder" fördern (Ehrenmitglieder waren Gustav Mahler und Ferruccio Busoni). Schönberg berichtete Posa von der Fron seiner Nebentätigkeit, die seine eigentliche Arbeit (damals das Streichquartett op. 7 und das Lied für Orchester op. 8/4) behindere:

Vielleicht komme ich aber doch noch dazu. Leider muss ich viel Fischof [sic! ${ }^{8}$ claviermäßig verschlucken, und orchestermäßig herausbrechen, wiedergeben! Ich habe kürzlich gesagt, wenn mir einmal Gedenktafeln an Landorten gesetzt werden müßten: „hier componierte er ...", so könnte es leider immer nur heißen: ,Hier instrumentierte er die Oper von Fischof [sic!] oder Holländer etc. Ich werde wohl einmal dadurch berühmt werden. Für meinen vorher erwähnten Ehrgeiz ein hohes Ziell [sic!] “๐

Die Liebe zur qualitativen populären Musik aber blieb und zieht sich durch das gesamte Werk, siehe die Walzerbearbeitungen Schönbergs, Bergs und Weberns für einen Abend des Vereins für musikalische Privataufführungen in Wien am 27. Mai 1921 oder die Bearbeitung der Drei Volkslieder für gemischten Chor a cappella op. 49 (1948). Sie findet sich auch in Werken der Zwölftontechnik, etwa der Suite für kleine Klarinette, Klarinette, Baßklarinette, Geige, Bratsche, Violoncello und Klavier op. 29 (1924-26) ${ }^{\mathrm{II}}$ oder

$7 \quad$ Ibid., 46.

8 Robert Fischhof (1856-1918), österreichischer Pianist und Komponist. Schönberg arbeitete damals an der Instrumentierung und dem Klavierauszug der Oper Der Bergkönig (UA Graz 1906).

Victor Hollaender (1866-1940), Operetten- und Revuekomponist, komponierte auch für das Überbrettl.

10 Brief vom 13. Juli 1904 aus Mödling.

11 Peter Andraschke, „Volkstümlichkeit (Länder, Walzer, Marsch) in der Wiener Klassik und in der Wiener Schule", in Mozart und Schönberg. Wiener Klassik und Wiener 
dem 4. Streichquartett op. 37 (1936). Und er beeinflußte damit auch viele seiner Schüler. Darauf wies Hanns Eisler in einem Gespräch mit Nathan Notowicz hin:

Übrigens, das ist wenig bekannt, Schönbergs Lieblingsmusik war zum Teil Wiener Lieder und auch Walzer von Johann Strauß. Die Begeisterung, mit der er uns den Johann Strauß erklärte - er hat übrigens einige Walzer auch bearbeitet - machte auf mich einen außerordentlichen Eindruck. Meine Vorliebe für Wiener Musik, aber auch andere Volksmusiken verdanke ich auch Schönberg. Denn als junger Mann in der Schönbergschen Schule pflegte ich natürlich diese Art von Musizieren von Heurigen-Liedern zu verachten. Erst Schönberg zeigte mir, was darin für Feinheiten sind.

Ich erinnere mich, wie er mir einmal erklärte, wie hübsch das sogenannte Fiakerlied harmonisiert sei. Da es sich in solchem tonalen Stück doch nur um einige Dreiklänge und deren Umkehrungen handelt, muß man schon ein großer Geist sein, um darin die Feinheiten zu sehen. Diese Art des Hörens scheint mir heute nicht so leicht vorzukommen, nämlich, daß man in den einfachsten Tonverbindungen Geschmack und Sicherheit beweist. Auch das konnte man von Schönberg lernen. ${ }^{\text {I2 }}$

Das Fiakerlied stammt von Gustav Pick (1832-1921), entstand zum 10ojährigen Bestehen der Fiakerzunft und ist dem Grabenfiaker gewidmet. Es wurde erstmals am 24. Mai 1885 von dem bekannten Alexander Girardi im Prater gesungen und ist eines der berühmtesten Wiener Lieder. Sein Refrain:

Mei Stolz is, i bin halt an echt's Weaner Kind, an Fiaker so wia man net alle Tag find't, und mei Bluat is so lüftig. so leicht wia da Wind, $i$ bin halt an echt's Weaner Kind.

Schule (= Schriften des Wissenschaftszentrums Arnold Schönberg 7), Hrsg. Hartmut Krones und Christian Meyer (Wien etc.: Böhlau, 2012), 29-56; Peter Andraschke, „Der volkstümliche Herder: Ausblicke in die Moderne. Eine musikalische Dokumentation", in Ideen und Ideale. Johann Gottfried Herder in Ost und West (= Rombach Wissenschaften. Reihe Litterae 103), Hrsg. Peter Andraschke und Helmut Loos (Freiburg i. Br.: Rombach, 2002), 167-207, darin: „3. Ännchen von Tharau“, 186-203.

Nathan Notowicz, Wir reden hier nicht von Napoleon. Wir reden von Ihnen! Gespräche mit Hanns Eisler und Gerhart Eisler, Hrsg. Jürgen Elsner (Berlin/DDR: Verlag Neue Musik, 1971), 47f. 
Schönberg hat dieses „Wiener Fiakerlied“ in seiner Jugend für zwei Violinen bearbeitet, das Autograph liegt in der Arnold Schoenberg - Hans Nachod Collection der Library of North Texas State University. ${ }^{13}$ Das Fiakerlied hat einen sentimental-nostalgischen Ausdruck, der für zahlreiche Wiener Lieder typisch ist.

Die ersten Vorarbeiten zu Schönbergs umfangreicher Schrift Der musikalische Gedanke reichen in die 1920er Jahre zurück, die endgültige Ausführung begann er im amerikanischen Exil in New York am 5. Juni 1934 in deutscher Sprache. Viele Beispiele von Märschen, Walzern und aus Operetten finden sich u. a. in dem Kapitel Populäre Musik und Melodik vom 3. Juli 1934. Es beginnt:

Die populäre Wirkung populärer Musik beruht auf ihrer breiten Verständlichkeit. Trotzdem aber unterscheiden echte Kenner (die es auch hier giebt) Originelles von Unoriginellem, Gutes von Schlechtem. ${ }^{\mathrm{I}}$

Die analysierten Beispiele sind: ${ }^{\text {IS }}$

Johann Strauß (Sohn): Lagunenwalzer op. 411

An der schönen blauen Donau op. 314

Rosen aus dem Süden op. 388

Sängerlust. Polka op. 328

Der Zigeunerbaron: „Wer uns getraut“ und „So voll von Fröhlichkeit"

Schatz-Walzer op. 418, Takt 24-40

Johann Strauß (Vater): Radetzkymarsch op. 228

W. A. Mozart: Die Zauberflöte KV 620, 1. Akt, „Der Vogelhändler bin ich ja“

Karl Komzák (Sohn): Erzherzog-Albrecht-Marsch op. 136

Franz Lehár: Die lustige Witwe, 3. Akt, „Lippen schweigen“,

Franz Schubert: 16 Deutsche und Zwei Ecossiasen für Klavier D 783

Veröffentlicht bei John A. Kimmey Jr., The Arnold Schoenberg - Hans Nachod Collection (= Detroit Studies in Music Bibliography 41) (Detroit, Information Coordinators, 1979), 182f; abgedruckt bei Andraschke, „Volkstümlichkeit“ (Anm. 10), $36 f$.

14 Arnold Schönberg, Der musikalische Gedanke (= Sämtliche Schriften. Kritische Gesamtausgabe. Abteilung II. Lehrwerke 6, Fragment gebliebene Lehrwerke I), Hrsg. Hartmut Krones (Wien: Universal Edition, 2018), 77. 
Joseph Lanner: Die Werber op. 103

Schrammel, Johann: s' Herz von an echtn Weana

Vindobona

Gustav Pick: Wiener Fiakerlied

Die entsprechenden Noten finden sich zum Teil noch in seiner Bibliothek im Arnold Schönberg Center in Wien. Interessant ist, daß Schönberg in den Beispielen nicht unterscheidet zwischen den gängigen Kategorien E-Musik und U-Musik, das Kriterium ist die Qualität.

Auf das Fiakerlied wurde bereits hingewiesen. Ausführlicher sei nun auf Lehárs Die lustige Witwe eingegangen. Noch heute wird von den Kindern Schönbergs tradiert, daß ihre Mutter Gertrud erzählte, der Vater habe diese Operette instrumentiert. ${ }^{16}$

\section{Franz Lehár}

Franz Lehár (1870, Komorn - 1948, Bad Ischl) wurde im damals österreich-ungarischen Komorn (heute Slowakei) geboren. Seine Muttersprache war ungarisch, bald kam deutsch hinzu, später weitere Sprachen. Er bekannte sich zeitlebens zum Ungartum und entschied sich 1919 für die ungarische Staatsbürgerschaft. Er behielt seine Unterschrift Lehár Ferenc, in der er seinen Vornamen zu einem stilisierten Violinschlüssel gestaltete, auch dann bei, wenn er zusätzlich mit dem deutschen Franz zeichnete. Lehárs Biographie zeigt gewisse Parallelen zu Schönberg, dessen Familie väterlicherseits auch aus Österreich-Ungarn stammte und der dann, da er zu Preßburg/Bratislava „zugehörig " war, tschechoslowakischer Staatsbürger wurde. ${ }^{17}$

Schönberg und Lehár kannten einander aus dem Wien der Jahrhundertwende, wo sie einander in Intellektuellen-Cafés begegneten, in denen Schönberg viele wichtige Vertreter der Operette kennenlernte. Er hatte, wie sein Biograph H. H. Stuckenschmidt schreibt,

16 Mitgeteilt von Therese Muxeneder.

17 Hartmut Krones, „Arnold Schönberg als ,Kind“ Österreich-Ungarns“, in Die Rezeption der Wiener Schule in Osteuropa (Musikgeschichte in Mittel- und Osteuropa. Mitteilungen der internationalen Arbeitsgemeinschaft an der Universität Leipzig 19), Hrsg. Hartmut Krones, Helmut Loos und Klaus-Peter Koch (Leipzig: Gudrun Schröder, 2017), 3-16. 
zeitlebens eine gewisse Zärtlichkeit für die Klassische Wiener Operette bewahrt, hörte sich gute Stücke von Franz Lehár gern an und übertrug diese Neigung auf einige seiner Schüler. ${ }^{18}$

Schönberg besaß auch über seine Familie Verbindungen zum Lehárkreis. So heiratete seine Cousine Malvina, die große Jugendliebe, Robert Bodansky, den Librettisten von Lehárs Operetten Der Graf von Luxemburg (1909) und Zigeunerliebe (1910).

\section{Lehárs 60. Geburtstag am 30. April 1930}

Mit Datum vom 18. April 1930 erreichte Schönberg eine Anfrage vom Neuen Wiener Journal:

Euer Hochwohlgeboren!

Franz Lehar, der Meister der Wiener Operette feiert in den nächsten Tagen seinen sechzigsten Geburtstag. Wir bitten Sie um eine kurze Aeusserung über Lehar und sein Schaffen, die wir anlässlich seines Geburtstages im Rahmen anderer Aeusserungen veröffentlichen wollen.

Mit bestem Dank für Ihre freundliche Mühewaltung, empfiehlt sich Ihr sehr ergebener

[hs. Unterschrift:] Dr. Walther Nagelstock

Chefredakteur-Stellvertreter

des

„Neuen Wiener Journal“

Auf der Rückseite des Schreibens konzipierte Schönberg Gedanken für eine Antwort. ${ }^{19}$ Die folgende Übertragung bringt einen fortlautenden Text, ohne Veränderung der Worte, aber mit wenigen Umstellungen und Übernahmen aus den Streichungen:

Es ist ein weitverbreiteter Irrtum, dass ein Musiker, der nicht genug Talent zu einer Oper hat, eine Operette schreiben kann:

Der schreibt eine schlechte. 
Auch zu einer Operette ist ein ganzes Talent erforderlich, und [...] bei Lehar finde ich diese vollkommene Uebereinstimmung zwischen dem Gedanken und Ausdrucks=Weise und Form des Ausdrucks.

Eine gute Operette muss an sich ebenso vollendet sein, wie eine gute Oper. Es ist möglich, daß auch die Operette einmal einen andern Weg gehen wird, aber jedenfalls ist Lehar unbekümmert den seinen mit der vollendeten Sicherheit wahren Könnens gegangen. Vielleicht zeigt sich das am deutlichsten in den Vorzeichen der neuen Oper im Rahmen des musikalischen Theaters neue musikalische Lebensbilder zu zeigen und eine neue Art von Humor.

Die österreichische Tageszeitung Neues Wiener Journal erschien von 1893 bis 31. Januar 1939. Sie verstand sich als unparteiisch und verfolgte keine klare politische Linie, war in der Grundtendenz antimarxistisch. Ihre Offenheit für zionistische Fragen hing damit zusammen, daß ihre Gründer und Herausgeber Juden waren: Jakob Lippowitz (1865, Leipzig - Wien, 1934 durch Freitod) und Oscar Loewenstein (1868, Danzig - 1939, London), der bis in die 1930er Jahre Chefredakteur war und 1939 mit seiner Frau nach England floh. Bekannte Mitarbeiter waren z. B. Egon Friedell und Hermann Bahr, der von 1916-1933 regelmäßig am Samstag seine Kolumne Tagebuch veröffentlichte. Nach dem Anschluß Österreichs vom 13. März 1938 wurde die Zeitung „arisiert“.

Die Antwort Schönbergs auf die Anfrage ist, falls er sie abgeliefert hat, nicht erschienen. In der Ausgabe vom 30. April findet sich auf den Seiten 4 und 5 einige Lehár gewidmete Spalten, und ins Auge fällt eine große Anzeige, die die Geschäftstüchtigkeit des Komponisten zeigt. Sie ist überschrieben mit Eine Lehár Ehrung, darunter ist eine große Abbildung des Geschenks - ein Ständer mit drei Flacons und der Erklärung: „Eine Widmung der über 220 Jahre alten Kölnischwasserfabrik, Farina Gegenüber ' ihrem treuen Anhänger Franz Lehár zum 6o. Geburtstag“. Der als „Meister Lehár" Titulierte dankte ausführlich und schloß:

[...] Wenn ich den heutigen Tag in voller Frische und Lebensfreude begehen kann, so danke ich dies nicht zuletzt auch Ihrem unvergleichlichen ,Farina Gegenüber' Kölnischwasser.

Wichtiger ist der Artikel von Dr. Fritz Löhner-Beda: Lehar nimmt Stellung. Zu seinem 6o. Geburtstag, in dem es um die Oper, den Jazz und den Tonfilm geht. Anton Freiherr v. Lehár titelte seinen Beitrag auf den Seite 5 und 6 Unsere Mutter. Meinem Bruder Franz zum 6o. Geburtstag gewidmet. 
Die Ehepaare Schönberg und Lehár trafen einander noch im Mai 1930 in Baden-Baden. Nach seiner Abreise schickte Schönberg aus Berlin, wo er damals wohnte, Lehár den Klavierauszug seiner neuen Oper Von heute auf morgen, der 1929 erschienen war (UA 1. Februar 1930 in Frankfurt am Main) mit der Widmung „dem verehrten, lieben Meister Lehár“. Es ist seine erste Oper in Zwölftontechnik, also eine anspruchsvolle Musik, von der er glaubte, daß sie den auch für die Moderne aufgeschlossenen Komponisten interessieren würde. Lehár, der noch in Baden-Baden kurte, bedankte sich per Telegramm vom 20. Mai:

fuer die liebe uebersendung ihrer juengsten hochinteressanten schoepfung herzlichen dank hatte riesenfreude wir muessen morgen leider direkt nach wien hoffen aber doch auf baldiges wiedersehen allerherzlichst getreuester $=$ franz lehar. ${ }^{20}$

\section{Die Operette und das Judentum}

1928 schrieb der katholische Ralph Benatzky, Komponist von Im weißen Rössl (1930), ein vielfach begabter Künstler, der in Wien zum Dr. phil. promoviert wurde, in sein Tagebuch über die Operettenszene:

Es gibt nur ein gutes, ideales Publikum: Die Juden! Sind viele im Raum, so wird gelacht, geweint, ist Wärme und Dankbarkeit da, Verständnis und Anerkennung und, sind viele Juden in einer Stadt, ist eben auch Publikum da. Die geistige Beweglichkeit dieser Rasse, die rasche Auffassung, die Kultur, der Sinn für die Pointe sind ideal für den Künstler, und nicht umsonst rekrutiert sich das Hauptkontingent der ausübenden und schaffenden Künstler aus dieser Nation. Von uns Wiener Komponisten z. B. Lehár, Strauß, Kálmán, Fall, Granichstädten [sic!], Eysler, Stolz, Engel-Berger, Erwin, Krauss, Werau, R. Fall, Katscher, Ch. Weinberger etc. sind bloß Lehár und ich Christen, von den Verlegern Wiens: Weinberger, Herzmansky, Hein, Call, Marischka bloß Herzmansky Christ, von den Librettisten kenne ich zur Zeit überhaupt keinen, der es wäre. Ebenso sind von den Schauspielern und Schauspielerinnen, Sängern und Sängerinnen und Tänzerinnen die Nichtjuden nur ganz selten, von den Direktoren gar nicht zu reden. ${ }^{21}$

20 Abgebildet bei Stuckenschmidt (Anm. 5), 307.

21 Ralph Benatzky, Triumph und Tristesse. Aus den Tagebüchern von 1919 bis 1949, Hrsg. Inge Jens und Christiane Niklew (Berlin: Parthos, 2002), 66. 
Die nationalsozialistische Kulturpolitik erkannte dieses Problem. So hielt der Reichsdramaturg und Berliner Oberregierungsrat Rainer Schlösser 1934 in einem Schreiben an den Reichspropagandaminister Joseph Goebbels fest: „Bei Machtübernahme war die Lage auf dem Operettenmarkt so, daß 80 Prozent der Produktion sowohl musikalisch wie textlich jüdischen Ursprungs war. ${ }^{\text {(222 }}$

Diese jüdische Dominanz reichte bis in die Frühzeit der Operette und über das deutsche Kulturgebiet hinaus. So war z. B. Jaques Offenbach Sohn eines jüdischen Kantors. Und diese Sachlage führte während des Naziregimes zu folgenreichen Repressalien gegen Personen aus dem Umkreis der Operette.

\section{Franz Lehár und das Naziregime}

Die Machtergreifung der Nationalsozialisten in Deutschland hatte auch für Lehár Konsequenzen. Er war seit 1924 mit der geschiedenen Sophie Meth, geb. Peschkis (1878-1947) verheiratet, einer Wiener Jüdin (Vater Sigmund, 1851-1918, Mutter Ernestine geb. Kohn); ihr erster Mann Heinrich Meth (1865-1942) ist im KZ Treblinka umgekommen. Vorgeworfen wurde Lehár aber auch seine häufige Zusammenarbeit mit nichtarischen Librettisten, Sängern und Theaterunternehmern und daß er in Wien vor allem in jüdischen Kreisen verkehrte. So waren auch die Librettisten der Operette Die lustige Witwe Juden, was wegen ihrer Pseudonyme leicht zu übersehen ist. Leo Stein, eigentlich Rosenstein, starb bereits 1921. Victor Léon, eigentlich Hirschfeld, war der Sohn eines Rabbiners, erhielt 1938 Berufsverbot, starb 1940 und ist auf dem Wiener Zentralfriedhof im Alten Israelitischen Teil begraben. Mit dem berühmten Tenor Richard Tauber war Lehár seit 1923 befreundet und schrieb für ihn eine Reihe von Rollen (u. a. im Land des Lächelns und in Giuditta). Der römisch-katholische Tauber wurde wegen seiner jüdischen Großeltern väterlicherseits 1933 von einem SA-Trupp in Berlin als Judenlümmel beschimpft sowie verprügelt und emigrierte schließlich 1938 nach Großbritannien. Er versuchte auch Lehár sehr eindringlich dazu zu bewegen.

Lehár lehnte ab, zum einen, da er kein Englisch konnte und dadurch für sich keine berufliche Zukunft im Ausland sah. Auch fühlte er sich in Österreich sicher durch seine offizielle Reputation, so war er z. B. seit 1920 Ehren-

22 Marina Maisel, „Dein ist mein ganzes Herz. Die Welt der Operette: Benefizveranstaltung für das Jüdische Zentrum am Jakobsplatz“, Jüdische Allgemeine, 16. 2. 2006, https://www.juedische-allgemeine.de/allgemein/dein-ist-mein-ganzes-herz/. 
mitglied der AKM und seit 1926 der Gesellschaft zur Hebung der Wiener Volkskunst. Zum anderen glaubte er, daß er als katholischer ungarischer Staatsbürger - Ungarn war damals ein wichtiger Verbündeter des Deutschen Reiches - kaum angreifbar wäre. Einen Höhepunkt in seiner Karriere bildete die Uraufführung seines letzten Bühnenwerks Giuditta am 20. Jänner 1934 an der Wiener Staatsoper gewesen, das von 120 Rundfunksendern live übertragen und vom Publikum gefeiert wurde. Mit dieser Musikalischen Komödie in fünf Bildern orientierte er sich an der veristischen Oper, die ihn stets initiiert hatte und zu der er hier tendierte; im Textbuch lautet die Gattung: Spieloper. Der vielfach begabte und tätige Fritz Löhner-Beda, u. a. Librettist von Giuditta, promovierter Jurist, ausgezeichneter Sportler, Gründungsmitglied und erster Präsident des jüdischen Wiener Sportvereins „Hakoah“, hatte sich trotz Warnungen wegen seiner Bekanntheit sicher gefühlt, wurde aber bereits am Tag des Einmarsches der deutschen Truppen in Österreich am 13. März 1938 festgenommen, nach Dachau, dann nach Buchenwald deportiert und schließlich 1942 in Auschwitz umgebracht. ${ }^{23}$

Trotz dieser Beispiele aus unmittelbarer Umgebung blieb Lehár und versuchte sich, so gut er konnte, zu arrangieren. ${ }^{24}$ Dabei erlebte er eine ständige Gratwanderung zwischen Hoffnung und Angst. Eine große Chance setzte Lehár auf seine künstlerischen Erfolge, vor allem durch Die lustige Witwe. Sie wurde am 30. Dezember 1905 im Theater an der Wien urauf-

23 Günther Schwarberg, Dein ist mein ganzes Herz. Die Geschichte des Fritz Löhner-Beda, der die schönsten Lieder der Welt schrieb, und warum Hitler ihn ermorden ließ (Göttingen: Steidl, 200o).

24 Eine redaktionelle Ergänzung (H. K.) soll zeigen, wie weit dieses „Arrangieren“ Lehárs ging: Die AKM, deren Ehrenpräsident Lehár war, wurde im August 1938 aufgelöst und in die deutsche STAGMA übergeführt. Bei der letzten Generalversammlung der AKM, bei der deren Auflösung und Überführung beschlossen wurde, unterstrich der Generaldirektor der STAGMA, Leo Ritter, laut dem Protokoll in seiner Ansprache vor allem folgendes: „Auch die nationalsozialistische Partei fordere nach strenger Pflichterfüllung des Tages am Abend Unterhaltung und Entspannung, ein Erfordernis, welchem gerade die ostmärkische Unterhaltungsmusik besonders Rechnung trage. Daher seien gerade die ostmärkischen Komponisten der [durchgestrichen: Partei, hs. darüber:] Stagma sehr willkommen. In Würdigung dieser Tatsache habe Herr Reichsminister Dr. Joseph Goebbels den Ehrenpräsidenten der staatlich genehmigten Gesellschaft der Autoren, Komponisten und Musikverleger (AKM) reg. Genossenschaft m.b.H., Meister Franz Lehar, in den Beirat der Stagma berufen als den führenden deutschen Unterhaltungskomponisten, ja sogar als den grössten überhaupt lebenden Unterhaltungskomponisten. (Lebhafter Beifall) Er freue sich, der Versammlung mitteilen zu können, dass Meister Franz Lehar dieser Berufung Folge geleistet habe [...].“ Zit. nach: Hartmut Krones, Das Ende der „alten“ A.K.M. (März 1938), die Gründung der „neuen“ AKM (Juni 1945) und die Folgen (Wien: AKM, 2014), 76. 
geführt. Trotz einer zunächst eher verhaltenen Resonanz war sie rasch erfolgreich und gehört bis heute zu den meistgespielten Werken des Komponisten. Adolf Hitler war bei der Uraufführung anwesend gewesen und blieb seitdem ein großer Verehrer Lehárs. Als die beiden einander 1936 bei einer Tagung in Berlin begegneten, war Hitler, nach Albert Speers Zeugnis, „noch Tage danach beglückt über dieses bedeutungsvolle Zusammentreffen ${ }^{\text {(2) }}$. Diese besondere Verbindung half, wurde aber von Teilen des Regimes, vor allem von Alfred Rosenberg, dem führenden Ideologen der NSDAP und seit 1933 Leiter des Außenpolitischen Amtes, unterlaufen, der Lehárs Vita und das seiner Umgebung sehr genau durchleuchtete. Ein erster Boykott von Lehárs Aufführungen wurde dank einer Intervention Goebbels rasch aufgehoben. 1938 wurde Lehárs Frau zur „Ehrenarierin“ erklärt, ${ }^{26} 1940$ erhielt er in Berlin bzw. Wien aus Hitlers Hand Auszeichnungen, darunter eine Goethemedaille $^{27}$, und das trotz eines damaligen Verbots seines Singspiels Friederike (UA Berlin 1928) wegen der jüdischen Librettisten. Allerdings wurde das Werk in Privataufführungen bei Goebbels gegeben. ${ }^{28}$

Lehár revanchierte sich verschiedentlich: So schenkte er Hitler 1938 zum Geburtstag ein in Maroquin-Leder gebundenes Bändchen zur Erinnerung an die 50. Aufführung der Lustigen Witwe; 1941 stellte er sich für Propagandakonzerte im besetzten Paris zur Verfügung. Und er ließ sich von den hohen Kulturfunktionären Peter Kreuder und Fritz Fischer überzeugen, seine Lustige Witwe als Revue in 33 Bildern für das Münchner Gärtnertheater bearbeiten zu lassen. Kreuder erinnert sich:

Ich fuhr zu Franz Lehár nach Bad Ischl und rang ihm die Erlaubnis ab, die "Lustige Witwe“ neu bearbeiten zu dürfen. Ich modernisierte seine Operette und polierte sie mit vielen Jazzelementen auf. Nach der rauschenden Premiere begaben wir uns alle wieder in das Künstlerhaus am Lenbachplatz. [...] Hitler erschien [...]. Er erzählte mir, daß er als junger Mann in Wien viele Aufführungen der "Lustigen Witwe“ gesehen hätte und von meiner Neubearbeitung restlos begeistert sei. Er nannte es nicht Jazz, was ich gespielt hatte. Er nannte es "moderne Rhythmen“.29

25 Maisel, „Dein ist mein ganzes Herz“ (Anm. 21).

26 Stefan Frey, Was sagt ihr zu diesem Erfolg. Franz Lehár und die Unterhaltungsmusik des 20. Jahrhunderts (Frankfurt am Main / Leipzig: Insel, 1999), $338 \mathrm{f}$.

29 Peter Kreuder, Nur Puppen haben keine Tränen. Ein Lebensbericht (Bergisch Gladbach: Bastei-Lübbe, 1973), 251. 
Trotzdem wurde wieder versucht, Lehárs Frau zu deportieren. ${ }^{30}$ Und noch Anfang Januar 1945, drei Monate vor der Befreiung Österreichs vom Nazi-Regime, wurde Lehár in Wien wegen seiner Weigerung, sich von seiner nichtarischen Frau zu trennen, unter Hausarrest gestellt.

\section{Bibliographie}

Andraschke, Peter. „Der volkstümliche Herder: Ausblicke in die Moderne. Eine musikalische Dokumentation". In Ideen und Ideale. Johann Gottfried Herder in Ost und West (= Rombach Wissenschaften. Reihe Litterae 103), hrsg. von Peter Andraschke und Helmut Loos, 167-207. Freiburg i. Br.: Rombach, 2002.

Andraschke, Peter. „Volkstümlichkeit (Länder, Walzer, Marsch) in der Wiener Klassik und in der Wiener Schule". In Mozart und Schönberg. Wiener Klassik und Wiener Schule (= Schriften des Wissenschaftszentrums Arnold Schönberg 7), hrsg. von Hartmut Krones und Christian Meyer, 29-56. Wien etc.: Böhlau, 2012.

Arnold Schönberg Center (schoenberg.at), Archiv - Briefe, ID 14612 (Rückseite). Benatzky, Ralph. Triumph und Tristesse. Aus den Tagebüchern von 1919 bis 1949, hrsg. von Inge Jens und Christiane Niklew. Berlin: Parthos, 2002.

Frey, Stefan. Was sagt ihr zu diesem Erfolg. Franz Lehár und die Unterhaltungsmusik des 2o. Jahrhunderts. Frankfurt am Main / Leipzig: Insel, 1999.

Grun, Bernard. Gold und Silber. Franz Lehár und seine Welt. München, Wien: Langen Müller, 1970.

Kimmey Jr., John A. The Arnold Schoenberg - Hans Nachod Collection (= Detroit Studies in Music Bibliography 41). Detroit, Information Coordinators, 1979.

Kreuder, Peter. Nur Puppen haben keine Tränen. Ein Lebensbericht. Bergisch Gladbach: Bastei-Lübbe, 1973.

Krones, Hartmut. Das Ende der "alten“ A.K.M. (März 1938), die Gründung der „neuen“ AKM (Juni 1945) und die Folgen. Wien: AKM, 2014.

Krones, Hartmut. „Arnold Schönberg als Kind“ Österreich-Ungarns“. In Die Rezeption der Wiener Schule in Osteuropa (Musikgeschichte in Mittelund Osteuropa. Mitteillungen der internationalen Arbeitsgemeinschaft an der Universität Leipzig 19), hrsg. von Hartmut Krones, Helmut Loos und Klaus-Peter Koch, 3-16. Leipzig: Gudrun Schröder, 2017.

30 Bernard Grun, Gold und Silber. Franz Lehár und seine Welt (München, Wien: Langen Müller, 1970), 291. 
Maisel, Marina. „Dein ist mein ganzes Herz. Die Welt der Operette: Benefizveranstaltung für das Jüdische Zentrum am Jakobsplatz“. Jüdische Allgemeine, 16. 2. 2006. https://www.juedische-allgemeine.de/allgemein/ dein-ist-mein-ganzes-herz/.

Muxeneder, Therese. „Walzer für Streichorchester (ca. 1897). Einführung“. In Salzburger Festspiele 2004. Programmheft der Uraufführung. Salzburg: s. n., August 2004 .

Notowicz, Nathan. Wir reden hier nicht von Napoleon. Wir reden von Ihnen! Gespräche mit Hanns Eisler und Gerhart Eisler, hrsg. von Jürgen Elsner. Berlin/DDR: Verlag Neue Musik, 1971.

Schönberg, Arnold. „Rückblick“. In Stil und Gedanke. Aufsätze zur Musik (Gesammelte Schriften 1), hrsg. von Ivan Vojtěch, 397-408. Frankfurt a. M.: Fischer, 1976.

Schönberg, Arnold. Der musikalische Gedanke (= Sämtliche Schriften. Kritische Gesamtausgabe. Abteilung II. Lehrwerke 6, Fragment gebliebene Lehrwerke I), hrsg. von Hartmut Krones. Wien: Universal Edition, 2018.

Schwarberg, Günther. Dein ist mein ganzes Herz. Die Geschichte des Fritz Löhner-Beda, der die schönsten Lieder der Welt schrieb, und warum Hitler ihn ermorden ließ. Göttingen: Steidl, 2000.

Stuckenschmidt, H. H. Schönberg. Leben. Umwelt. Werk. Zürich, Freiburg: Atlantis, 1974. 